\title{
Bohuslav Diviš
}

Über Gitterpunkte in mehrdimensionalen Ellipsoiden

Czechoslovak Mathematical Journal, Vol. 20 (1970), No. 1, 130-139

Persistent URL: http://dml.cz/dmlcz/100952

\section{Terms of use:}

(C) Institute of Mathematics AS CR, 1970

Institute of Mathematics of the Czech Academy of Sciences provides access to digitized documents strictly for personal use. Each copy of any part of this document must contain these Terms of use.

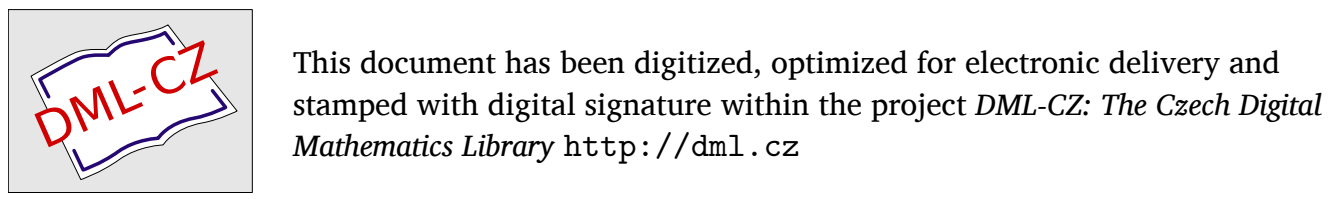




\title{
ÜBER GITTERPUNKTE IN MEHRDIMENSIONALEN ELLIPSOIDEN
}

\author{
Bohuslav Diviš, Praha
}

(Eingelangt am 4. April 1969)

Es sei $\sigma$ ganz, $\sigma \geqq 2$; weiter seien $r_{j} \geqq 1$, ganz $(j=1,2, \ldots, \sigma)$. In dieser Abhandlung betrachten wir ausschließlich quadratische Formen der Gestalt

$$
Q(u)=\sum_{j=1}^{\sigma} \alpha_{j}\left(u_{1, j}^{2}+u_{2, j}^{2}+\ldots+u_{r_{j}, j}^{2}\right), \quad \alpha_{j}>0 \quad(j=1,2, \ldots, \sigma) .
$$

Bezeichnen wir mit $A_{Q}(x)$ die Anzahl der Gitterpunkte im abgeschlossenen Ellipsoid $Q(u) \leqq x$, d. h.

$$
A_{Q}(x)=\sum_{Q(u) \leqq x} 1
$$

Mit $V_{Q}(x)$ bezeichnen wir den Inhalt dieses Ellipsoides und setzen

$$
P_{Q}(x)=A_{Q}(x)-V_{Q}(x) .
$$

Für jede gegebene Form $Q$ bedeute $f(Q)$ die untere Grenze derjenigen $\omega$, für welche

$$
P_{Q}(x)=O\left(x^{\omega}\right)
$$

ist. Mit anderen Worten: wenn wir kurz $f$ statt $f(Q)$ schreiben, für jedes $\varepsilon>0$ ist

$$
P_{Q}(x)=O\left(x^{f+\varepsilon}\right) ; \quad P_{Q}(x)=\Omega\left(x^{f-\varepsilon}\right) .
$$

Um das Hauptergebnis dieser Arbeit ausdrücken zu können, ist es notwendig, noch eine Bezeichnung einzuführen. Es sei $k \geqq 1$, ganz, $\delta_{j}$ seien reell $(j=1,2, \ldots, k)$. Mit $\beta\left(\delta_{1}, \delta_{2}, \ldots, \delta_{k}\right)$ bezeichnen wir die obere Grenze derjenigen $\omega$, für welche das System der Ungleichungen

$$
\left|\delta_{j}-\frac{p_{j}}{q}\right|<\frac{1}{q^{\omega}} \quad(j=1,2, \ldots, k)
$$

unendlich viele Lösungen mit ganzzahligen $(k+1)$-Tupeln $\left\{p_{1 n}, p_{2 n}, \ldots, p_{k n} ; q_{n}\right\}_{n=1}^{\infty}$, $q_{n} \rightarrow+\infty$ hat. In der vorliegenden Arbeit beweisen wir folgenden 
Satz 1. Es sei $\sigma \geqq 2$, ganz, $\alpha_{j}>0(j=1,2, \ldots, \sigma)$; sei $\beta=\beta\left(\alpha_{2} / \alpha_{1}, \alpha_{3} / \alpha_{1}, \ldots\right.$ $\left.\ldots, \alpha_{\sigma} / \alpha_{1}\right)$. Weiter seien $r_{j} \geqq 2 \beta /(\beta-1)$, ganz $(j=1,2, \ldots, \sigma), r=r_{1}+r_{2}+\ldots$ $\ldots+r_{\sigma}$. Dann ist für die Formen der Gestalt (1)

$$
f(Q)=\frac{r}{2}-1-\frac{1}{\beta-1}
$$

Dabei setzen wir für $\beta=+\infty: 1 /(\beta-1)=0,2 \beta /(\beta-1)=2$. Diese Behauptung war schon früher mit einer ziemlich ausführlichen Einleitung in die Problematik ohne Beweis veröffentlicht (vgl. [3]). Die Kenntnis der eben zitierten Arbeit ist empfehlenswert, jedoch nicht erforderlich. Zum Beweis wurde die von V. JARNíK in den unten zitierten Arbeiten ausgearbeitete Methode benutzt. Zunächst wollen wir unsere Aufgabe etwas anders formulieren und gleichzeitig vereinfachen. Nach (2) ist unser Satz mit diesen zwei Behauptungen gleichbedeutend: für jedes $\varepsilon>0$ ist es

$$
\begin{gathered}
P_{Q}(x)=O\left(x^{r / 2-1-1 /(\beta-1)+\varepsilon}\right), \\
P_{Q}(x)=\Omega\left(x^{r / 2-1-1 /(\beta-1)-\varepsilon}\right) .
\end{gathered}
$$

Behauptung (4) ist bekannt (vgl. [2]). Weiter ist der Satz 1 im Falle $\beta=+\infty$ auch bekannt (vgl. [1]). Statt des Satzes 1 genügt es also folgende Behauptung zu beweisen:

Satz 2. Es sei $\sigma \geqq 2$, ganz, $\alpha_{j}>0(j=1,2, \ldots, \sigma)$; sei $\beta=\beta\left(\alpha_{2} / \alpha_{1}, \alpha_{3} / \alpha_{1}, \ldots\right.$ $\left.\ldots, \alpha_{\sigma} / \alpha_{1}\right)$. Weiter seien $r_{j} \geqq 2 \beta /(\beta-1)$, ganz $(j=1,2, \ldots, \sigma), r=r_{1}+r_{2}+\ldots$ $\ldots+r_{\sigma}$. Überdies sei $\beta<\gamma<+\infty$. Dann ist für die Formen der Gestalt (1) für jedes $\varepsilon>0$

$$
P_{Q}(x)=O\left(x^{r / 2-1-1 /(\gamma-1)+\varepsilon}\right) .
$$

Zunächst beweisen wir zwei Hilfssätze.

Hilfssatz 1. Setzen wir $\|\zeta\|=\underset{p \operatorname{Manz}}{\operatorname{Min}}|\zeta-p|$ für reelle $\zeta$. Weiter sei $k \geqq 1$, ganz; $\delta_{j}(1 \leqq j \leqq k)$ seien reell, sei $\beta=\beta\left(\delta_{1}, \delta_{2}, \ldots, \delta_{k}\right), \beta<\gamma<+\infty$. L und $M$ seien positiv. Dann ist die Anzahl $v$ der ganzen Zahlen z, welche den Ungleichungen

$$
1 \leqq z \leqq M, \quad\left\|z \delta_{j}\right\|<L \quad(j=1,2, \ldots, k)
$$

genügen, höchstens gleich $c L^{1 /(\gamma-1)} M$, wobei die Konstante c nur von $\delta_{j}(j=1,2, \ldots$ ...,k) und $\gamma$ abhängt.

Beweis. Ohne Einschränkung der Allgemeinheit sei $v \geqq 1, L<\frac{1}{4}$ und seien $1 \leqq$ $\leqq z_{1}<z_{2}<\ldots<z_{v} \leqq M$ diejenigen ganzen Zahlen, für welche (5) gilt. Bezeichnen wir mit $\omega$ die kleinste natürliche Zahl, so daß $\left\|\omega \delta_{j}\right\|<2 L(j=1,2, \ldots, k)$ ist. Bemerken wir, daß $\left\|\zeta_{1} \pm \zeta_{2}\right\| \leqq\left\|\zeta_{1}\right\|+\left\|\zeta_{2}\right\|$ für alle reellen $\zeta_{1}, \zeta_{2}$ ist. Es ist dann $z_{1} \geqq \omega, z_{2}-z_{1} \geqq \omega, z_{3}-z_{2} \geqq \omega, \ldots, z_{v}-z_{v-1} \geqq \omega$, also $z_{v} \geqq v \omega$. Weiter folgt 
aus der Definition von $\beta$ und $\gamma$ die Existenz eines solchen $j_{0}, 1 \leqq j_{0} \leqq k$, so da $\beta$ $\left\|\omega \delta_{j 0}\right\| \geqq c_{1} / \omega^{\gamma-1}$ ist, wobei die Konstante $c_{1}$ nur von $\delta_{j}(j=1,2, \ldots, k)$ und $\gamma$ abhängt. Nach der Definition von $\omega$ haben wir also

$$
2 L>\left\|\omega \delta_{j 0}\right\| \geqq \frac{c_{1}}{\omega^{\gamma-1}} \geqq c_{1}\left(\frac{v}{z_{v}}\right)^{\gamma-1} \geqq c_{1}\left(\frac{v}{M}\right)^{\gamma-1},
$$

woraus folgt

$$
v \leqq\left(\frac{2}{c_{1}}\right)^{1 /(\gamma-1)} L^{1 /(\gamma-1)} M
$$

Schreiben wir $c$ statt $\left(2 / c_{1}\right)^{1 /(\gamma-1)}$, so ist der Hilfssatz bewiesen.

Hilfssatz 2. Es sei $\sigma \geqq 2$, ganz, $\alpha_{j}>0,(j=1,2, \ldots, \sigma), \beta=\beta\left(\alpha_{2} / \alpha_{1}, \alpha_{3} / \alpha_{1}, \ldots\right.$ $\left.\ldots, \alpha_{\sigma} / \alpha_{1}\right), \beta<\gamma<+\infty$. Weiter sei $D>0, x>1, \varepsilon>0$. Schließlich seien $l_{,} m_{j}$ $(j=1,2, \ldots, \sigma), n_{j}(j=2,3, \ldots, \sigma)$ ganze nichtnegative Zahlen, $2^{m_{j}} \leqq \sqrt{ } x(j=$ $=1,2, \ldots, \sigma)$. Dann ist die Anzahl der ganzzahligen Systeme $\left\{h_{1}, k_{1} ; h_{2}, k_{2} ; \ldots\right.$; $\left.h_{\sigma}, k_{\sigma}\right\}$, welche den Ungleichungen

$$
\begin{gathered}
2^{n_{\sigma}} k_{\sigma} \leqq \ldots \leqq 2^{n_{3}} k_{3} \leqq 2^{n_{2}} k_{2}, \\
\left|\frac{h_{1}}{k_{1}} \frac{1}{\alpha_{1}}-\frac{h_{j}}{k_{j}} \frac{1}{\alpha_{j}}\right|<\frac{D}{2^{n_{j}} k_{j} \sqrt{ } x}(j=2,3, \ldots, \sigma), \\
2^{l} \leqq h_{1}<2^{l+1}, \quad 2^{m_{j}} \leqq k_{j}<2^{m_{j}+1} \quad(j=1,2, \ldots, \sigma)
\end{gathered}
$$

genügen, höchstens gleich

$$
\left.d(\varepsilon) 2^{\left(l+m_{2}+m_{3}+\ldots+m_{\sigma}\right)(1+\varepsilon)+\left(m_{1}+m_{2}+\ldots+m_{\sigma-1}-n_{\sigma}\right) /(\gamma-1)} x^{-1 / 2(\gamma-1)} \cdot \cdot^{1}\right)
$$

Beweis. Aus den Ungleichungen (7) folgt

$$
\left.\left|h_{1} k_{j} \frac{\alpha_{j}}{\alpha_{1}}-h_{j} k_{1}\right|<d \frac{k_{1}}{2^{n_{j}} \sqrt{ } x}<d \frac{k_{1} k_{j}}{2^{n_{\sigma}} k_{\sigma} \sqrt{ } x} \quad(j=2,3, \ldots, \sigma),{ }^{2}\right)
$$

woraus wir erhalten

$$
\left|h_{1} \prod_{\mu=2}^{\sigma} k_{\mu} \frac{\alpha_{j}}{\alpha_{1}}-h_{j} \prod_{\substack{\mu=1 \\ \mu \neq j}}^{\sigma} k_{\mu}\right|<d \frac{\prod_{\mu=1}^{\sigma-1} k_{\mu}}{2^{n_{\sigma}} \sqrt{ } x}<d 2^{m_{1}+m_{2}+\ldots+m_{\sigma-1}-n_{\sigma}} x^{-1 / 2}
$$

\footnotetext{
1) Mit $d(\varepsilon)$ bezeichnen wir unterschiedslos positive Konstanten, die nur von $\sigma, \alpha_{1}, \alpha_{2}, \ldots$ $\ldots, \alpha_{\sigma}, \gamma, D, \varepsilon$ abhängen.

$\left.{ }^{2}\right)$ Mit $d$ bezeichnen wir unterschiedslos positive Konstanten, die nur von $\sigma, \alpha_{1}, \alpha_{2}, \ldots$ $\ldots, \alpha_{\sigma}, \gamma, D$ abhängen.
} 
für $j=2,3, \ldots, \sigma$. Es ist also

$$
\left\|h_{1} k_{2} k_{3} \ldots k_{\sigma} \frac{\alpha_{j}}{\alpha_{1}}\right\|<d 2^{m_{1}+m_{2}+\ldots+m_{\sigma-1}-n_{\sigma}} x^{-1 / 2} \quad(j=2,3, \ldots, \sigma) .
$$

Nach dem Hilfssatz 1 kommt es mit Hinsicht auf $h_{1} k_{2} k_{3} \ldots k_{\sigma}<2^{\sigma} .2^{l+m_{2}+m_{3}+\ldots+m_{\sigma}}$ für das Produkt $h_{1} k_{2} k_{3} \ldots k_{\sigma}$ höchstens

$$
d 2^{l+m_{2}+m_{3}+\ldots+m_{\sigma}+\left(m_{1}+m_{2}+\ldots+m_{\sigma-1}-n_{\sigma}\right) /(\gamma-1)} x^{-1 / 2(\gamma-1)}
$$

Werte in Betracht. Wegen (9) und mit Hinsicht auf $d k_{1} / 2^{n_{j}} \sqrt{ } x<d\left(\operatorname{denn} 2^{m_{1}} \leqq \sqrt{ } x\right)$ entspricht jedem Wert des Produktes $h_{1} k_{j}$ höchstens $d$ Werte für das Produkt $h_{j} k_{1}$ und es ist $h_{j} k_{1}<d 2^{l+m_{j}}(j=2,3, \ldots, \sigma)$. Weil die Anzahl der Teiler einer ganzen Zahl $X$ für jedes $\eta>0$ gleich $O\left(X^{\eta}\right)$ ist, haben wir bei gegebenem $h_{1} k_{2} k_{3} \ldots k_{\sigma}$ für das Produkt $h_{1} k_{j}$ höchstens $d(\eta) 2^{\left(l+m_{2}+m_{3}+\ldots+m_{\sigma}\right) \eta}$ Möglichkeiten $(j=2,3, \ldots, \sigma)$, also höchstens $d(\eta) 2^{\left(l+m_{2}+m_{3}+\ldots+m_{\sigma}\right) \eta}$ Möglichkeiten für das Produkt $h_{j} k_{1}(j=$ $=2,3, \ldots, \sigma)$. Bei gegebenem $h_{1} k_{j}$ haben wir höchstens $d(\eta) 2^{\left(l+m_{2}+\ldots+m_{\sigma}\right) \eta}$ Möglichkeiten für $h_{1}, k_{j}(j=2,3, \ldots, \sigma)$, bei gegebenem $h_{j} k_{1}$ haben wir höchstens $d(\eta)$. $.2^{\left(l+m_{2}+m_{3}+\ldots+m_{\sigma}\right) \eta}$ Möglichkeiten für $k_{1}, h_{j}(j=2,3, \ldots, \sigma)$. Wir haben also bei gegebenem $h_{1} k_{2} k_{3} \ldots k_{\sigma}$ höchstens $d(\eta) 2^{\left(l+m_{2}+m_{3}+\ldots+m_{\sigma}\right) 4 \sigma \eta}$ Möglichkeiten für die Anzahl der Systeme $\left\{h_{1}, k_{1} ; h_{2}, k_{2} ; \ldots ; h_{\sigma}, k_{\sigma}\right\}$. Schreiben wir $\varepsilon$ statt $4 \sigma \eta$, so ist der Hilfssatz bewiesen.

Beweis des Satzes 2. $\sigma, r_{1}, r_{2}, \ldots, r_{\sigma}, r, \alpha_{1}, \alpha_{2}, \ldots, \alpha_{\sigma}, \beta, Q(u), A_{Q}(x), V_{Q}(x), P_{Q}(x)$ mögen die im Satz 1 definierte Bedeutung haben. Sei $\beta<+\infty, \beta<\gamma<+\infty$. Wir setzen $\theta(s)=\sum_{m=-\infty}^{+\infty} \mathrm{e}^{-m^{2} s}$ für $\operatorname{Re} s>0$,

$$
A=\operatorname{Max}_{1 \leqq j \leqq \sigma} \frac{2 \pi}{\alpha_{j}}, \quad z=x^{-1 /(\beta-1)}, \quad s=\frac{1}{x}+i t \quad(t \text { reell }) .
$$

Dann gilt (vgl. [2], §2)

$$
P_{Q}(x)=O\left(x^{r / 4}+x^{r / 2-1} z+\frac{1}{z} \int_{A / \sqrt{ } x}^{\infty}\left|\theta^{r_{1}}\left(\alpha_{1} s\right) \ldots \theta^{r_{\sigma}}\left(\alpha_{\sigma} s\right)\right| \operatorname{Min}(1, t z) \frac{\mathrm{d} t}{t^{2}}\right) .
$$

Man kann sich leicht davon überzeugen, daß unter unseren Voraussetzungen immer $\frac{1}{2} r-1-1 /(\beta-1) \geqq \frac{1}{4} r$ ist. Es ist nämlich immer $\beta \geqq \sigma /(\sigma-1)$. Daraus folgt, daß es folgendes zu beweisen genügt: für jedes $\varepsilon>0$ ist

$$
\frac{1}{z} \int_{A / \sqrt{ } x}^{\infty}\left|\theta^{r_{1}}\left(\alpha_{1} s\right) \ldots \theta^{r_{\sigma}}\left(\alpha_{\sigma} s\right)\right| \operatorname{Min}(1, t z) \frac{\mathrm{d} t}{t^{2}}=O\left(x^{r / 2-1-1 /(\gamma-1)+\varepsilon}\right)\left(s=\frac{1}{x}+i t\right) .
$$

Wir legen jetzt bei gegebenem $x>1$ auf das Intervall $-\infty<t<+\infty$ alle FareyBrüche $h / k$, wobei $h \geqq 0,0<k \leqq \sqrt{ } x,(h, k)=1$ und konstruieren noch ihre Me- 
dianten, d. h. alle Zahlen $(h+\bar{h}) /(k+\bar{k})$, wo $h / k, \bar{h} / \bar{k}$ zwei benachbarte unter unseren Farey-Brüchen sind. Wir bezeichnen mit $B_{h, k}$ das linksseitig abgeschlossene, rechtsseitig offene Intervall, dessen Endpunkte zwei benachbarte Medianten sind und welches den Punkt $h / k$ enthält. Diese Intervalle überdecken dann lückenlos die ganze reelle Achse und sind paarweise punktfremd. Jetzt ist folgendes bekannt (vgl. [1]): Wenn $t$ im Intervall $\left.\left(2 \pi / \alpha_{j}\right) B_{h, k}{ }^{3}\right)$ liegt, dann ist

$$
\left.\left|\theta\left(\alpha_{j} s\right)\right|<\frac{c}{\sqrt{ } k} \operatorname{Min}\left(\sqrt{ } x, \frac{1}{t-\frac{2 \pi}{\alpha_{j}} \frac{h}{k}}\right)^{4}\right)\left(\operatorname{Min}\left(a, \frac{1}{0}\right)=a\right) .
$$

Bekanntlich ist weiter

$$
B_{h, k} \subset\left\langle\frac{h}{k}-\frac{1}{k \sqrt{ } x}, \frac{h}{k}+\frac{1}{k \sqrt{ } x}\right) .
$$

$\mathrm{Zu}$ jedem $t$ unseres Integrationsintervalles $\langle A| \sqrt{ } x, \infty)$ gibt es jetzt eindeutig $2 \sigma$ Zahlen $h_{1}, k_{1}, \ldots, h_{\sigma}, k_{\sigma}$, so daß $t$ im Durchschnitt der $\sigma$ Intervalle $\left(2 \pi / \alpha_{1}\right) B_{h_{1}, k_{1}}, \ldots$ $\ldots,\left(2 \pi / \alpha_{\sigma}\right) B_{h_{\sigma}, k_{\sigma}}$ liegt. Wenn $t$ im Durchschnitt der Intervalle $\left(2 \pi / \alpha_{1}\right) B_{h_{1}, k_{1}}, \ldots$ $\ldots,\left(2 \pi / \alpha_{\sigma}\right) B_{h_{\sigma}, k_{\sigma}}$ liegt, dann gibt es nach (14) genau ein System der ganzen nichtnegativen Zahlen $\left(n_{1}, n_{2}, \ldots, n_{\sigma}\right)$, so daß

$$
\frac{1}{2^{n_{j}+1} k_{j} \sqrt{ } x}<\left|\frac{\alpha_{j}}{2 \pi} t-\frac{h_{j}}{k_{j}}\right| \leqq \frac{1}{2^{n_{j}} k_{j} \sqrt{ } x} \quad(j=1,2, \ldots, \sigma)
$$

ist, mit Ausnahme der Punkte $t=2 \pi h_{j} \mid \alpha_{j} k_{j}(j=1,2, \ldots, \sigma)$.

Wir definieren jetzt abzählbar viele Mengen $\mathfrak{M}(h, k, n)=\mathfrak{M}\left(h_{1}, k_{1}, n_{1} ; \ldots\right.$ $\left.\ldots ; h_{\sigma}, k_{\sigma}, n_{\sigma}\right)$ folgenderweise: wenn $h_{1}, \ldots, h_{\sigma}, k_{1}, \ldots, k_{\sigma}, n_{1}, \ldots, n_{\sigma}$ ganze Zahlen sind, so daß $h_{j}>0,0<k_{j} \leqq \sqrt{ } x,\left(h_{j}, k_{j}\right)=1, n_{j} \geqq 0(j=1,2, \ldots, \sigma)$, sei $\mathfrak{M}(h, k, n)$ Menge derjenigen $t$, welche im Durchschnitt der Intervalle $\left(2 \pi / \alpha_{j}\right) B_{h_{j}, k_{j}}(j=1,2, \ldots$ $\ldots, \sigma),\langle A| \sqrt{ } x, \infty)$ liegen und die Ungleichungen (15) erfüllen. Die Mengen $\mathfrak{M}(h, k, n)$ sind paarweise disjunkt und überdecken das ganze Intervall $\langle A / \sqrt{ } x, \infty)$ mit Ausnahme abzählbar vieler Punkte $t=2 \pi h_{j} / \alpha_{j} k_{j}(j=1,2, \ldots, \sigma)$. Um (12) zu beweisen, genügt es also zu zeigen, daß für jedes $\varepsilon>0$ (es genügt hinreichend klein)

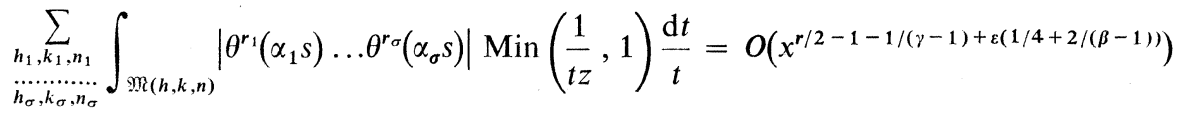
mit $s=1 / x+i t$ ist.

\footnotetext{
${ }^{3}$ ) Wenn $I=\left\langle a_{1}, a_{2}\right)$ und $\left.a_{3}\right\rangle 0$, dann bedeute $a_{3} I$ das Intervall $\left\langle a_{1} a_{3}, a_{2} a_{3}\right)$.

$\left.{ }^{4}\right)$ Mit $c$ bezeichnen wir unterschiedslos positive Konstanten, welche nur von $\sigma, \alpha_{1}, \alpha_{2}, \ldots$ $\ldots, \alpha_{\sigma}, r_{1}, r_{2}, \ldots, r_{\sigma}, \gamma$ abhängen.
} 
Aus Symmetriegründen beschränken wir uns dabei auf jene $\mathfrak{M}(h, k, n)$, für welche

$$
2^{n_{1}} k_{1} \geqq 2^{n_{2}} k_{2} \geqq \ldots \geqq 2^{n_{\sigma}} k_{\sigma}
$$

gilt. Diese Mengen $\mathfrak{M}(h, k, n)$ werden wir weiter folgendermaßen klassifizieren. Wir sagen, daß die Menge $\mathfrak{M}(h, k, n)$ zur Klasse $\mathfrak{R}(l, m, n)\left(l, m_{j}, n_{j}\right.$ ganz, nichtnegativ) gehört, wenn $2^{l} \leqq h_{1}<2^{l+1}, 2^{m_{j}} \leqq k_{j}<2^{m_{j}+1}(j=1,2, \ldots, \sigma)$ ist. Jede Menge $\mathfrak{M}(h, k, n)$ gehört genau zu einer Klasse $\mathfrak{R}(l, m, n)$. Dabei können wir uns nur auf jene $\mathfrak{R}(l, m, n)$ beschränken, für welche $2^{m_{j}} \leqq \sqrt{ } x(j=1,2, \ldots, \sigma)$ ist. Soll die Menge $\mathfrak{M}(h, k, n)$ der Klasse $\mathfrak{R}(l, m, n)$ nichtleer sein, so muß nach (15) und wegen

$$
\left|\frac{h_{1}}{k_{1}} \frac{1}{\alpha_{1}}-\frac{h_{j}}{k_{j}} \frac{1}{\alpha_{j}}\right|<\frac{c}{2^{n_{j}} k_{j} \sqrt{ } x} \quad(j=2,3, \ldots, \sigma)
$$

gelten. Nach dem Hilfssatz 2 ist die Anzahl der nichtleeren Mengen $\mathfrak{M}(h, k, n)$ der Klasse $\mathfrak{\Omega}(l, m, n)$ für jedes $\varepsilon>0$ höchstens gleich

$$
\left.c(\varepsilon) 2^{\left(l+m_{2}+m_{3}+\ldots+m_{\sigma}\right)(1+\varepsilon)+\left(m_{1}+m_{2}+\ldots+m_{\sigma-1}-n_{\sigma}\right) /(\gamma-1)} x^{-1 / 2(\gamma-1)} \cdot{ }^{5}\right)
$$

Wir bemerken noch:

1. Ist $\mathfrak{M}(h, k, n)$ eine nichtleere Menge der Klasse $\mathfrak{R}(l, m, n)$, dann ist ihr Maß wegen (15) höchstens gleich $c / 2^{m_{1}+n_{1}} \sqrt{ } x$.

2. Ist $t \in \mathfrak{M}(h, k, n)$ der Klasse $\mathfrak{\Re}(l, m, n)$, dann ist nach (13), (15)

$$
\left|\theta\left(\alpha_{j} s\right)\right|<\frac{c}{\sqrt{ } 2^{m_{j}}} \operatorname{Min}\left(\sqrt{ } x, \sqrt{ } 2^{m_{j}+n_{j}} \sqrt[4]{x}\right) \quad(1 \leqq j \leqq \sigma)
$$

3. Wenn $t$ in der Menge $\mathfrak{M}(h, k, n)$ der Klasse $\mathfrak{i}(l, m, n)$ liegt, dann ist wegen (14) $\left|\left(\alpha_{1} / 2 \pi\right) t-h_{1}\right| k_{1} \mid \leqq 1 / k_{1} \sqrt{ } x$, also $c 2^{l-m_{1}}<t<c 2^{l-m_{1}}$ für $x>c$.

4. Die Anzahl der nichtleeren Mengen $\mathfrak{M}(h, k, n)$ der Klasse $\mathfrak{i}(l, m, n)$ haben wir nach oben mit (19) abgeschätzt.

Es sei jetzt $\varepsilon$ mit $0<\varepsilon<\operatorname{Min}\left(\frac{1}{2}\right.$, Min $\left.\frac{1}{4}\left(r_{j}-2 \gamma /(\gamma-1)\right)\right)$ gegeben und wir untersuchen den linken Ausdruck in (16). Zunächst schätzen wir:

$$
\operatorname{Min}\left(\frac{1}{t z}, 1\right) \leqq\left(\frac{1}{t z}\right)^{2 \varepsilon}=x^{2 \varepsilon /(\beta-1)} \frac{1}{t^{2 \varepsilon}} \text { für } \varepsilon<\frac{1}{2}
$$

\footnotetext{
$\left.{ }^{5}\right)$ Mit $c(\varepsilon)$ bezeichnen wir unterschiedslos positive Konstanten, die nur von $\sigma, \alpha_{1}, \alpha_{2}, \ldots$ $\ldots, \alpha_{\sigma}, r_{1}, r_{2}, \ldots, r_{\sigma}, \gamma, \varepsilon$ abhängen.
} 
Weiter schätzen wir (vgl. die Bemerkungen 1.-4.)

$$
\begin{aligned}
& \sum_{\substack{h_{1}, k_{1}, n_{1} \\
\vdots \\
h_{\sigma}, k_{\sigma}, n_{\sigma}}} \int_{\mathfrak{M}(h, k, n)}\left|\theta^{r_{1}}\left(\alpha_{1} s\right) \ldots \theta^{r_{\sigma}}\left(\alpha_{\sigma} s\right)\right| \frac{\mathrm{d} t}{t^{1+2 \varepsilon}} \leqq c(\varepsilon) \sum_{\substack{l, m_{1}, n_{1} \\
\vdots \\
m_{\sigma}, n_{\sigma}}} \frac{2^{m_{1}(1+2 \varepsilon)}}{2^{l(1+2 \varepsilon)}} \frac{1}{2^{m_{1}+n_{1}} \sqrt{ } x} \times \\
& \times 2^{\left(l+m_{2}+\ldots+m_{\sigma}\right)(1+\varepsilon)+\left(m_{1}+\ldots+m_{\sigma}-1-n_{\sigma}\right) /(\gamma-1)} x^{-1 / 2(\gamma-1)} \prod_{j=1}^{\sigma} \frac{x^{r_{j} / 4}}{2^{m_{j} r_{j} / 2}} \times \\
& \times \operatorname{Min}\left(x^{r_{j} / 4}, 2^{m_{j} r_{j} / 2+n_{j} r_{j} / 2}\right) \leqq c(\varepsilon) x^{r / 4-1 / 2-1 / 2(\gamma-1)} \sum_{m_{1}, n_{1}} 2^{m_{1}\left(1 /(\gamma-1)+2 \varepsilon-r_{1} / 2\right)-n_{1}} \times \\
& \times \operatorname{Min}\left(x^{r_{1} / 4}, 2^{m_{1} r_{1} / 2+n_{1} r_{1} / 2}\right) \prod_{j=2}^{\sigma-1} \sum_{m_{j}, n_{j}} 2^{m_{j}\left(1+\varepsilon+1 /(\gamma-1)-r_{j} / 2\right)} \operatorname{Min}\left(x^{r_{j} / 4}, 2^{m_{j} r_{j} / 2+n_{j} r_{j} / 2}\right) \times \\
& \times \sum_{m_{\sigma}, n_{\sigma}} 2^{m_{\sigma}\left(1+\varepsilon-r_{\sigma} / 2\right)-n_{\sigma} /(\gamma-1)} \operatorname{Min}\left(x^{r_{\sigma} / 4}, 2^{m_{\sigma} r_{\sigma} / 2+n_{\sigma} r_{\sigma} / 2}\right)
\end{aligned}
$$

Dabei ist wegen (17) und mit Hinsicht auf $2^{m_{j}} \leqq k_{j}<2^{m_{j}+1}(j=1,2, \ldots, \sigma)$ über solche $m_{1}, n_{1}, \ldots, m_{\sigma}, n_{\sigma}$ zu summieren, für welche

$$
m_{1}+n_{1} \geqq m_{2}+n_{2} \geqq \ldots \geqq m_{\sigma}+n_{\sigma}, \quad 2^{m_{j}} \leqq \sqrt{ } x \quad(j=1,2, \ldots, \sigma)
$$

ist. Folglich haben wir

$$
\begin{gathered}
\sum_{2^{m_{\sigma}+n_{\sigma} \leqq \sqrt{ } x}} 2^{m_{\sigma}\left(1+\varepsilon-r_{\sigma} / 2\right)-n_{\sigma} /(\gamma-1)} \operatorname{Min}\left(x^{r_{\sigma} / 4}, 2^{m_{\sigma} r_{\sigma} / 2+n_{\sigma} r_{\sigma} / 2}\right) \leqq \\
\sum_{2^{m_{\sigma}+n_{\sigma}} \leqq \sqrt{ } x} 2^{m_{\sigma}(1+\varepsilon)+n_{\sigma}\left(r_{\sigma} / 2-1 /(\gamma-1)\right)} \leqq c(\varepsilon) \sum_{2^{n_{\sigma}} \leqq \sqrt{ } x}\left(\frac{\sqrt{ } x}{2^{n_{\sigma}}}\right)^{1+\varepsilon} 2^{n_{\sigma}\left(r_{\sigma} / 2-1 /(\gamma-1)\right)} \leqq \\
\leqq c(\varepsilon) x^{1 / 2+\varepsilon / 2} \sum_{2^{n_{\sigma}} \leqq \sqrt{ } x} 2^{n_{\sigma}\left(r_{\sigma} / 2-1-1 /(\gamma-1)-\varepsilon\right)} \leqq c(\varepsilon) x^{r_{\sigma} / 4-1 / 2(\gamma-1)},
\end{gathered}
$$

als

$$
\varepsilon<\frac{r_{\sigma}}{2}-\frac{\gamma}{\gamma-1}
$$

ist.

$$
\begin{aligned}
& \sum_{2^{m_{\sigma}+n_{\sigma}>\sqrt{ } x}} 2^{m_{\sigma}\left(1+\varepsilon-r_{\sigma} / 2\right)-n_{\sigma} /(\gamma-1)} \operatorname{Min}\left(x^{r_{\sigma} / 4}, 2^{m_{\sigma} r_{\sigma} / 2+n_{\sigma} r_{\sigma} / 2}\right) \leqq \\
& \leqq x^{r_{\sigma} / 4} \sum_{2^{m_{\sigma}+n_{\sigma}>\sqrt{ } x}} 2^{m_{\sigma}\left(1+\varepsilon-r_{\sigma} / 2\right)-n_{\sigma} /(\gamma-1)} \leqq c x^{r_{\sigma} / 4} \sum_{2^{m_{\sigma}} \leqq \sqrt{ } x} 2^{m_{\sigma}\left(1+\varepsilon-\left(r_{\sigma} / 2\right)\right)}\left(\frac{2^{m_{\sigma}}}{\sqrt{ } x}\right)^{1 /(\gamma-1)} \leqq \\
& \leqq c x^{r_{\sigma} / 4-1 / 2(\gamma-1)} \sum_{2^{m_{\sigma}} \leqq \sqrt{ } x} 2^{m_{\sigma}\left(1+\varepsilon-r_{\sigma} / 2+1 /(\gamma-1)\right)} \leqq c(\varepsilon) x^{r_{\sigma} / 4-1 / 2(\gamma-1)},
\end{aligned}
$$


als

$$
\varepsilon<\frac{r_{\sigma}}{2}-\frac{\gamma}{\gamma-1}
$$

ist.

Jetzt sei $2 \leqq j \leqq \sigma-1$. Wir erhalten (vgl. (21))

$$
\begin{gathered}
\sum_{2^{m_{j}+n_{j}} \leqq \sqrt{ } x} 2^{m_{j}\left(1+\varepsilon+1 /(\gamma-1)-r_{j} / 2\right)} \operatorname{Min}\left(x^{r_{j} / 4}, 2^{m_{j} r_{j} / 2+n_{j} r_{j} / 2}\right) \leqq \\
\leqq \sum_{2^{m_{j}+n_{j}} \leqq \sqrt{ } x} 2^{m_{j}(1+\varepsilon+1 /(\gamma-1))+n_{j} r_{j} / 2} \leqq c \sum_{2^{m_{j}} \leqq \sqrt{ } x} 2^{m_{j}(1+\varepsilon+1 /(\gamma-1))}\left(\frac{\sqrt{ } x}{2^{m_{j}}}\right)^{r_{j} / 2} \leqq \\
\leqq c x^{r_{j} / 4} \sum_{2^{m_{j}} \leqq \sqrt{ } x} 2^{m_{j}\left(1+\varepsilon+1 /(\gamma-1)-r_{j} / 2\right)} \leqq c(\varepsilon) x^{r_{j} / 4},
\end{gathered}
$$

als

$$
\varepsilon<\frac{r_{j}}{2}-\frac{\gamma}{\gamma-1}
$$

ist.

$$
\begin{gathered}
\sum_{2^{m_{j}+n_{j}>\sqrt{ } x}} 2^{m_{j}\left(1+\varepsilon+1 /(\gamma-1)-r_{j} / 2\right)} \operatorname{Min}\left(x^{r_{j} / 4}, 2^{m_{j} r_{j} / 2+n_{j} r_{j} / 2}\right) \leqq \\
\leqq x^{r_{j} / 4} \sum_{2^{m_{j}+n_{j}>\sqrt{ } x}} 2^{m_{j}\left(1+\varepsilon+1 /(\gamma-1)-r_{j} / 2\right)} \leqq c(\varepsilon) x^{r_{j} / 4} \sum_{n_{j} \leqq m_{1}+n_{1}} 1 \leqq c(\varepsilon) x^{r_{j} / 4}\left(m_{1}+n_{1}\right),
\end{gathered}
$$

als

$$
\varepsilon<\frac{r_{j}}{2}-\frac{\gamma}{\gamma-1}
$$

ist.

Schließlich haben wir

$$
\begin{gathered}
\sum_{2^{m_{1}+n_{1}} \leqq \sqrt{ } x} 2^{m_{1}\left(1 /(\gamma-1)+2 \varepsilon-r_{1} / 2\right)-n_{1}} \operatorname{Min}\left(x^{r_{1} / 4}, 2^{m_{1} r_{1} / 2+n_{1} r_{1} / 2}\right) \leqq \\
\leqq \sum_{2^{m_{1}+n_{1}} \leqq \sqrt{ } x} 2^{m_{1}(1 /(\gamma-1)+2 \varepsilon)+n_{1}\left(r_{1} / 2-1\right)} \leqq c(\varepsilon) \sum_{2^{n_{1}} \leqq \sqrt{ } x}\left(\frac{\sqrt{ } x}{2^{n_{1}}}\right)^{1 /(\gamma-1)+2 \varepsilon} 2^{n_{1}\left(r_{1} / 2-1\right)} \leqq \\
\leqq c(\varepsilon) x^{1 / 2(\gamma-1)+\varepsilon} \sum_{2^{n_{1}} \leqq \sqrt{ } x} 2^{n_{1}\left(r_{1} / 2-1-1 /(\gamma-1)-2 \varepsilon\right)} \leqq c(\varepsilon) x^{r_{1} / 4-1 / 2},
\end{gathered}
$$

als

$$
\varepsilon<\frac{1}{2}\left(\frac{r_{1}}{2}-\frac{\gamma}{\gamma-1}\right)
$$

ist. 


$$
\begin{aligned}
& \sum_{2^{m_{1}+n_{1}>\sqrt{ } x}} 2^{m_{1}\left(1 /(\gamma-1)+2 \varepsilon-r_{1} / 2\right)-n_{1}} \operatorname{Min}\left(x^{r_{1} / 4}, 2^{m_{1} r_{1} / 2+n_{1} r_{1} / 2}\right)\left(m_{1}+n_{1}\right)^{\sigma} \leqq \\
& \leqq x^{r_{1} / 4} \sum_{2^{m_{1}+n_{1}>\sqrt{ } x}} 2^{m_{1}\left(1 /(\gamma-1)+2 \varepsilon-r_{1} / 2\right)-n_{1}}\left(m_{1}+n_{1}\right)^{\sigma} \leqq \\
& \leqq c(\varepsilon) x^{r_{1} / 4} \sum_{2^{m_{1}+n_{1}}>\sqrt{ } x} 2^{m_{1}\left(1 /(\gamma-1)+2 \varepsilon-r_{1} / 2\right)-n_{1}} 2^{\left(m_{1}+n_{1}\right) \varepsilon / 2} \leqq \\
& \leqq c(\varepsilon) x^{r_{1} / 4} \sum_{2^{m_{1}} \leqq \sqrt{ } x} 2^{m_{1}\left(1 /(\gamma-1)-r_{1} / 2+5 \varepsilon / 2\right)}\left(\frac{2^{m_{1}}}{\sqrt{ } x}\right)^{1-\varepsilon / 2} \leqq \\
& \leqq c(\varepsilon) x^{r_{1} / 4-1 / 2+\varepsilon / 4} \sum_{2^{m_{1}} \leqq \sqrt{ } x} 2^{m_{1}\left(\gamma /(\gamma-1)+2 \varepsilon-r_{1} / 2\right)} \leqq c(\varepsilon) x^{r_{1} / 4-1 / 2+\varepsilon / 4},
\end{aligned}
$$

als

$$
\varepsilon<\operatorname{Min}\left(2, \frac{1}{4}\left(\frac{r_{1}}{2}-\frac{\gamma}{\gamma-1}\right)\right)
$$

ist.

Aus unseren Abschätzungen folgt, daß der linke Ausdruck in (16) höchstens gleich $($ vgl. (20))

$$
\begin{gathered}
c(\varepsilon) \exp \left\{\left(\frac{r}{4}-\frac{1}{2}-\frac{1}{2(\gamma-1)}+\frac{r_{\sigma}}{4}-\frac{1}{2(\gamma-1)}+\frac{r_{\sigma-1}}{4}+\right.\right. \\
\left.\left.+\ldots+\frac{r_{2}}{4}+\frac{r_{1}}{4}-\frac{1}{2}+\varepsilon\left(\frac{1}{4}+\frac{2}{\beta-1}\right)\right) \lg x\right\} \leqq \\
\leqq c(\varepsilon) x^{r / 2-1-1 /(\gamma-1)+\varepsilon(1 / 4+2(\beta-1))},
\end{gathered}
$$

als

$$
\varepsilon<\operatorname{Min}\left(\frac{1}{2}, \operatorname{Min}_{1 \leqq j \leqq \sigma} \frac{1}{4}\left(r_{j}-\frac{2 \gamma}{\gamma-1}\right)\right)
$$

ist, was zu beweisen war.

Durch geringe Modifikation des eben durchgeführten Beweises bekommen wir den

Satz 3. Es sei $\sigma \geqq 2$, ganz, weiter seien $r_{j} \geqq 1$, ganz $(j=1,2, \ldots, \sigma), r=$ $=r_{1}+r_{2}+\ldots+r_{\sigma}, \alpha_{j}>0(j=1,2, \ldots, \sigma), \beta\left(\alpha_{2} / \alpha_{1}, \alpha_{3} / \alpha_{1}, \ldots, \alpha_{\sigma} / \alpha_{1}\right)=\beta$. Dann gilt für jedes $\varepsilon>0$

$$
P_{Q}(x)=O\left(\exp \left\{\left(\frac{r}{2}-\frac{1}{4} \sum_{j=1}^{\sigma} \operatorname{Min}\left(\frac{2 \beta}{\beta-1}, r_{j}\right)+\left(\frac{\sigma}{2}-1\right) \frac{\beta}{\beta-1}+\varepsilon\right) \lg x\right\}\right)
$$

für die Formen der Gestalt (1).

Der Satz 3 ist zwar auch für $2 \leqq r \leqq 4$ richtig, aber in diesen Fällen sind schärfere Resultate bekannt. 
Alle Ergebnisse samt Beweisen lassen sich unmittelbar auf etwas allgemeinere Formen der Gestalt

$$
Q(u)=\sum_{j=1}^{\sigma} \alpha_{j} Q_{j}\left(u_{1, j}, u_{2, j}, \ldots, u_{r_{j}, j}\right) \quad\left(\alpha_{j}>0\right) \quad(j=1,2, \ldots, \sigma)
$$

übertragen, wo die quadratischen Formen $Q_{j}(j=1,2, \ldots, \sigma)$, positiv definit sind, und ganzzahlige Koeffizienten haben.

\section{Literaturverzeichnis}

[1] V. Jarnik: „Über Gitterpunkte in mehrdimensionalen Ellipsoiden“, Math. Annalen 100 (1928), 699-721.

[2] V. Jarnik: „Über Gitterpunkte in mehrdimensionalen Ellipsoiden: eine Anwendung des Hausdorffschen Maßbegriffes", Math. Zeitschrift 38 (1934), 217-256.

[3] B. Divis: "On lattice points in high-dimensional ellipsoids" (preliminary communication), Comment. Math. Univ. Carolinae, Vol. 9, fasc. 2, 199-205 (1968).

Anschrift des Verfassers: Praha 1, Žitná 25, ČSSR (Matematický ústav ČSAV). 